\title{
Testosterone Deprivation Aggravates Left-Ventricular Dysfunction in Male Obese Insulin-Resistant Rats via Impairing Cardiac Mitochondrial Function and Dynamics Proteins
}

\author{
Nattayaporn Apaiajai ${ }^{a, b}$ Titikorn Chunchai ${ }^{a-c}$ Thidarat Jaiwongkam ${ }^{a, b}$ \\ Sasiwan Kerdphoo ${ }^{a, b}$ Siriporn C. Chattipakorn ${ }^{a, b, d}$ Nipon Chattipakorn ${ }^{a-c}$ \\ ${ }^{a}$ Cardiac Electrophysiology Research and Training Center, Faculty of Medicine, Chiang Mai University, \\ Chiang Mai, Thailand; ${ }^{\mathrm{b} C e n t e r}$ of Excellence in Cardiac Electrophysiology Research, Chiang Mai University, \\ Chiang Mai, Thailand; ' Cardiac Electrophysiology Unit, Department of Physiology, Faculty of Medicine, \\ Chiang Mai University, Chiang Mai, Thailand; ' Department of Oral Biology and Diagnostic Sciences, Faculty of \\ Dentistry, Chiang Mai University, Chiang Mai, Thailand
}

\section{Keywords}

Testosterone deprivation · Obesity-related insulin resistance $\cdot$ Cardiac function - Mitochondrial function . Mitochondrial dynamics proteins

\footnotetext{
Abstract

Background: We have previously reported that testosterone deprivation at a very young age accelerated, but did not aggravate, left-ventricular (LV) dysfunction in obese insulin-resistant rats. However, the effects of testosterone deprivation during adulthood on LV function in obese insulin-resistant rats remains unclear. We hypothesized that testosterone deprivation aggravates LV dysfunction and cardiac autonomic imbalance via the impairment of cardiac mitochondrial function and dynamics proteins, a reduction in insulin receptor function, and an increase in apoptosis in obese insulin-resistant rats. Methods: Male rats were fed on either a normal diet (ND) or a high-fat diet (HFD) for 12 weeks. They were then subdivided into 2 groups: sham operation (NDS, HFS) and orchiectomy (NDO, HFO). Metabolic
}

parameters, blood pressure, heart rate variability (HRV), and LV function were determined at baseline and before and after orchiectomy. Mitochondrial function and dynamics proteins, insulin signaling, and apoptosis were determined 12 weeks postoperatively. Results: HFS rats exhibited obese insulin resistance, depressed HRV, and LV dysfunction. In HFO rats, systolic blood pressure was increased with more excessive depression of HRV and increased LV dysfunction, compared with HFS rats. These adverse cardiac effects were consistent with markedly increased mitochondrial dysfunction, reduced mitochondrial complex I and III proteins, reduced mitochondrial fusion proteins, and increased apoptosis, compared with HFS rats. However, testosterone deprivation did not lead to any alteration in the insulin-resistant condition in HFO rats, compared with HFS rats. Conclusion: We concluded that testosterone deprivation during adulthood aggravated the impairment of mitochondrial function, mitochondrial respiratory complex, mitochondrial dynamics proteins, and apoptosis, leading to LV dysfunction in obese insulin-resistant rats.

(c) 2018 S. Karger AG, Basel

\section{KARGER}

(c) 2018 S. Karger AG, Basel

E-Mail karger@karger.com

www.karger.com/ger
Nipon Chattipakorn, MD, PhD

Cardiac Electrophysiology Research and Training Center

Faculty of Medicine, Chiang Mai University

110 Intawaroros Rd., Sriphum, Muang, Chiang Mai 50200 (Thailand)

E-Mail nchattip@gmail.com 


\section{Introduction}

Both obesity-related insulin resistance and testosterone deprivation are known to increase the risk of cardiovascular disease [1], and in both human [2] and animal studies have been shown to be associated with left-ventricular (LV) dysfunction and cardiac autonomic imbalance [3-5]. Previous reports suggested that these adverse cardiac effects are due to mitochondrial dysfunction $[4,6]$. Mitochondria are highly mobile organelles, and their function is controlled by mitochondrial dynamics $[7,8]$. Changes in the fission/fusion balance in mitochondria can lead to mitochondrial dysfunction and apoptosis $[9,10]$. Mitochondrial fission is regulated by dynamin-related protein 1 (Drp1). Mitochondrial fusion is regulated by mitofusin2 (Mfn2) and optic atrophy1 (OPA1), these proteins being responsible for outer and inner mitochondrial membrane fusion, respectively [7]. It has been shown that Drp1 protein levels were increased whereas Mfn2 protein levels were decreased in testosterone-deprived rats with myocardial infarction, compared with the levels in rats with myocardial infarction alone [8].

Although testosterone levels usually decline with age, obesity is also associated with a reduction of testosterone level [11]. Our previous study using obese insulin-resistant rats demonstrated that testosterone deprivation at a very young age followed by long-term consumption of a high-fat diet (HFD) could accelerate but not aggravate LV dysfunction [4]. A recent study showed that obese insulin-resistant rats subjected to testosterone deprivation in middle age had an exacerbated insulin-resistant condition as indicated by increased HOMA-IR [12]. In addition, LV mass was also reduced and serum CK-MB levels were increased in testosterone-deprived obese insulin-resistant rats subjected to myocardial ischemia reperfusion injury [13]. Currently, the process involved in the alteration of cardiometabolic function in testosterone-deprived obese insulin-resistant rats remains unknown. Furthermore, the underlying mechanism responsible for the changes in LV function in testosterone-deprived obese insulin-resistant rats is still unclear. We hypothesized that testosterone deprivation aggravates LV dysfunction and cardiac autonomic imbalance via the impairment of cardiac mitochondrial function and mitochondrial dynamics, reduced myocardial insulin receptor function, and increased apoptosis in obese insulin-resistant rats.

\section{Materials and Methods}

\section{Ethical Approval}

All experiments in this study were approved by the Faculty of Medicine, Chiang Mai University Institutional Animal Care and Use Committee (permit No. 15/2559), in compliance with NIH guidelines and in accordance with the ARRIVE guidelines for reporting animal research.

\section{Animals and Experimental Design}

Thirty-two male Wistar rats (6 weeks old; 150-180 g) were obtained from Nomura Siam International Co., Ltd. (Bangkok, Thailand). The rats were housed in a room with controlled temperature and humidity, and were allowed access to water and food ad libitum. After 1 week of acclimatization, the rats were randomly divided into 2 dietary groups: a normal diet group (ND, a diet containing $19.77 \%$ energy from fat) and an HFD group (a diet containing $52.98 \%$ energy from fat). The rats were given their assigned diet for 12 weeks [14]. Then, the rats in each dietary group were randomly subdivided into 2 subgroups: a sham operation group $(n=$ 16; NDS: $n=8$ and HFS: $n=8$ ) and a bilateral orchiectomy (ORX) group ( $n=16$; NDO: $n=8$ and HFO: $n=8)$. Metabolic parameters, blood pressure, cardiac autonomic balance, and LV function were determined at baseline, after 12 weeks of HFD feeding, and 1, 2, 4, 8 , and 12 weeks postoperatively. LV function was determined invasively at 12 weeks postoperatively. Insulin (10 IU/kg; Eli Lilly and Company, IA, USA) was injected afterwards to stimulate myocardial insulin signaling [15]. Thirty minutes after the insulin injection, the rats were euthanized using Zoletil $100(150 \mathrm{mg} / \mathrm{kg}$; Virbac, Bangkok, Thailand) and Xylazine (9 mg/kg; LBS Labs, Bangkok, Thailand) via intramuscular injection, followed by decapitation, and the heart was rapidly excised to enable the determination of cardiac mitochondrial function, mitochondrial dynamics, myocardial insulin signaling, and apoptotic protein expression. The experimental protocol is shown in online supplementary Figure 1 (for all online suppl. material, see www.karger. com/doi/10.1159/000487188).

\section{Bilateral ORX}

Rats were anesthetized and maintained using $2 \%$ isoflurane with $98 \%$ oxygen. The ORX was performed under sterile conditions, the incision being made in the scrotal area [4]. The blood vessels and vas deferens were permanently ligated with 5-0 silk sutures, and the testes were then removed. The skin incision was closed using a nonabsorbable suture. The rats were carefully monitored to prevent them from chewing their sutures and to record any other complications. An analgesic drug ( $4 \mathrm{mg} / \mathrm{kg}$, $4 \%$ tolfenamic acid; Vetoquinol, France), and antibiotics ( $2 \mathrm{mg} / \mathrm{kg}, 2 \% \mathrm{mar}-$ bofloxacin; Vetoquinol) were administered via subcutaneous injection for 3 days after surgery [4].

\section{Determination of Metabolic Parameters}

Body weight and food intake were recorded weekly, and visceral fat weight was recorded after euthanasia. Blood was collected from the tail vein and centrifuged at $6,000 \mathrm{rpm}$ for $10 \mathrm{~min}$. The plasma was collected to allow the determination of plasma testosterone, insulin, glucose, triglyceride, total cholesterol, and HDL levels [16].

Plasma testosterone levels were determined using the ELISA technique at the Central Laboratory Service of Maharaj Nakorn
Apaiajai/Chunchai/Jaiwongkam/ Kerdphoo/Chattipakorn/Chattipakorn 
Chiang Mai Hospital (Faculty of Medicine, Chiang Mai University) [4]. Plasma insulin levels were determined using a commercial ELISA kit (Merck Millipore, MA, USA). Plasma glucose, total cholesterol, and triglyceride levels were determined using commercial colorimetric kits (Erba Mannheim, Mannheim, Germany). The degree of insulin resistance was assessed by the HOMA index, which was calculated from fasting plasma insulin and fasting plasma glucose concentration. Plasma HDL levels were determined using a commercial colorimetric kit (BioVision Inc., CA, USA). Plasma LDL levels were calculated using the Friedewald formula [16].

\section{Blood Pressure Determination}

Systolic blood pressure (SBP) and diastolic blood pressure (DBP) were determined in conscious rats using a noninvasive volume-pressure recording tail cuff method (CODA-2; Kent Scientific, CT, USA) [16].

\section{Heart Rate Variability Determination}

A frequency domain of heart rate variability (HRV) including a high-frequency (HF) and low-frequency (LF) band were used to represent cardiac autonomic balance for a short-term recording [17]. A lead II electrocardiogram (ECG) was recorded in conscious rats. The ECG signals were recorded through a signal transducer (PowerLab 4/25T; AD Instruments, Sydney, NSW, Australia), and operated through the Chart 5.0 program (AD Instruments). Power spectra of RR intervals were obtained using the fast Fourier transform algorithm. Both the LF $(0.2-0.6 \mathrm{~Hz})$ and $\mathrm{HF}(0.6-3 \mathrm{~Hz})$ bands were detected where LF represents sympathetic tone and HF represents parasympathetic tone. Therefore, an increased LF/HF ratio indicated cardiac sympathovagal imbalance [16].

\section{Function Determination}

LV function was determined noninvasively using echocardiography at baseline, after 12 weeks of HFD feeding, at 1, 2, 4, 8, and 12 weeks postoperatively. The rats were anesthetized using $1 \%$ isoflurane. An S12 probe was placed on the chest at the parasternal short axis, and connected to the machine (GE Vivid-i; GE Healthcare, IL, USA). The M-mode echocardiogram was recorded at the papillary muscle, and the percentage of LV ejection fraction (\%LVEF) was determined [16].

At 12 weeks postoperatively, an invasive LV function test was carried out using pressure-volume loop analysis. All rats were anesthetized using a combination of $50 \mathrm{mg} / \mathrm{kg}$ Zoletil (Virbac) and $3 \mathrm{mg} / \mathrm{kg}$ Xylazine (LBS Labs) via intramuscular injection. In each case the right carotid artery was identified, and a pressure-volume admittance catheter (1.9F VSL catheter; Transonic, NY, USA) was inserted through the right carotid artery and advanced into the LV. Then, the pressure-volume catheter was connected to the pressure-volume admittance system (ADV500; Transonic), and the signals were recorded via a Labscribe 2 program (iWorx, NH, USA). LV function including heart rate (HR), end-systolic and diastolic pressure (ESP, EDP), $\pm \mathrm{dP} / \mathrm{dt}$, end-systolic and diastolic volume (ESV, EDV), and stroke volume (SV) were determined [18].

\section{Cardiac Mitochondrial Function}

The heart tissue was minced and homogenized in an ice-cold buffer. Cardiac mitochondria were obtained using a differential centrifugation technique as previously described [19]. For cardiac mitochondrial ROS level determination, $0.4 \mathrm{mg} / \mathrm{mL}$ of cardiac mi- tochondrial protein was incubated with $2 \mu \mathrm{M}$ DCFH-DA dye at $25^{\circ} \mathrm{C}$ for $20 \mathrm{~min}$. The fluorescence intensity of DCF was detected at $\lambda$ ex $485 \mathrm{~nm}$ and $\lambda$ em $530 \mathrm{~nm}$ using a fluorescence microplate reader (BioTek, VT, USA). Increased DCF fluorescence intensity indicates increased cardiac mitochondrial ROS levels. For cardiac mitochondrial membrane potential (MMP) changes, $0.4 \mathrm{mg} / \mathrm{mL}$ of cardiac mitochondrial protein was incubated with $5 \mu \mathrm{M} \mathrm{JC}-1$ dye. JC- 1 is a ratiometric dye that exhibits potential-dependent accumulation in mitochondria. The red fluorescence intensity of JC-1 (J-aggregates) was detected at $\lambda$ ex $485 \mathrm{~nm}$ and $\lambda$ em $590 \mathrm{~nm}$, and it indicates an intact MMP or healthy mitochondria. The green fluorescence intensity of JC-1 (J-monomers) was detected at $\lambda$ ex $485 \mathrm{~nm}$ and $\lambda \mathrm{em} 530 \mathrm{~nm}$, and it indicates low MMP or unhealthy mitochondria. Both red and green fluorescence intensity were measured by a fluorescence microplate reader (BioTek). Therefore, a decreased red/green fluorescent intensity ratio indicates mitochondrial membrane depolarization [18].

For cardiac mitochondrial function, cardiac mitochondrial respiration was assessed using a high-throughput automated 96-well extracellular flux analyzer (XFe96; Agilent Seahorse, CA, USA); 0.5 $\mathrm{mg} / \mathrm{mL}$ of cardiac mitochondrial protein was used, and mitochondria were suspended in a medium buffer containing $100 \mathrm{mM} \mathrm{KCl}$, $10 \mathrm{mM} \mathrm{HEPES}, 5 \mathrm{mM} \mathrm{KH}_{2} \mathrm{PO}_{4}$, and $\mathrm{pH} 7.2$ in the presence of $0.2 \%$ fatty acid-free BSA. The XFe96 plate was coated with polyethylenimine (1:15,000 dilution) and incubated overnight at $37^{\circ} \mathrm{C}$, and the polyethylenimine was removed on the day of the assay; $20 \mu \mathrm{L}$ of $0.5 \mathrm{mg} / \mathrm{mL}$ of isolated cardiac mitochondria was loaded into the XFe96 plate, and mitochondria were centrifuged at 3,000 $g$ for 7 $\min$ at $4{ }^{\circ} \mathrm{C}$. Then, the mitochondria were incubated for $30 \mathrm{~min}$ at $37^{\circ} \mathrm{C}$ before starting the assay. First, pyruvate/malate (final concentration: $5 \mathrm{~mm}$ each) was added as NADH-linked substrate, followed by ADP (final concentration: $1 \mathrm{mM}$ ) administration to determine mitochondrial state 3 respiration. State 4 respiration was measured after adding oligomycin (final concentration: $1 \mu \mathrm{M}$ ) $[20,21]$.

\section{Plasma and Cardiac Malondialdehyde Determination}

In this study, cardiac tissue homogenates were used to determine cardiac malondialdehyde (MDA) levels as an indicator for cardiac oxidative stress. Plasma was used to determine plasma MDA levels as an indicator for circulating oxidative stress. These samples were mixed with $0.6 \%$ thiobarbituric acid (TBA) and 0.44 $\mathrm{M} \mathrm{H}_{3} \mathrm{PO}_{4}$, and then heated at $90^{\circ} \mathrm{C}$ for $30 \mathrm{~min}$ to become pinkcolored products called thiobarbituric acid reactive substances (TBARS). Plasma and cardiac TBARS concentration were determined directly from the standard curves using the HPLC system, and reported as MDA equivalent concentration. Plasma MDA concentration was expressed in $\mu \mathrm{M}$, and cardiac MDA concentration was expressed in $\mu \mathrm{M} / \mathrm{mg}$ protein $[4,19]$.

\section{Western Blot Analysis}

Sixty micrograms of the total protein were mixed with a loading buffer as previously described [16]. The protein was loaded onto 10 or $12.5 \%$ SDS-acrylamide gels, separated by electrophoresis, and then transferred onto $45-\mu \mathrm{m}$ nitrocellulose membrane (GE healthcare, Buckinghamshire, UK) in a wet-tank blotting system (Bio-Rad, CA, USA). The membranes were blocked in 5\% skim milk or $5 \%$ BSA in TBST.

For mitochondrial dynamic and respiratory chain complex determination, membranes were incubated with primary antibodies 


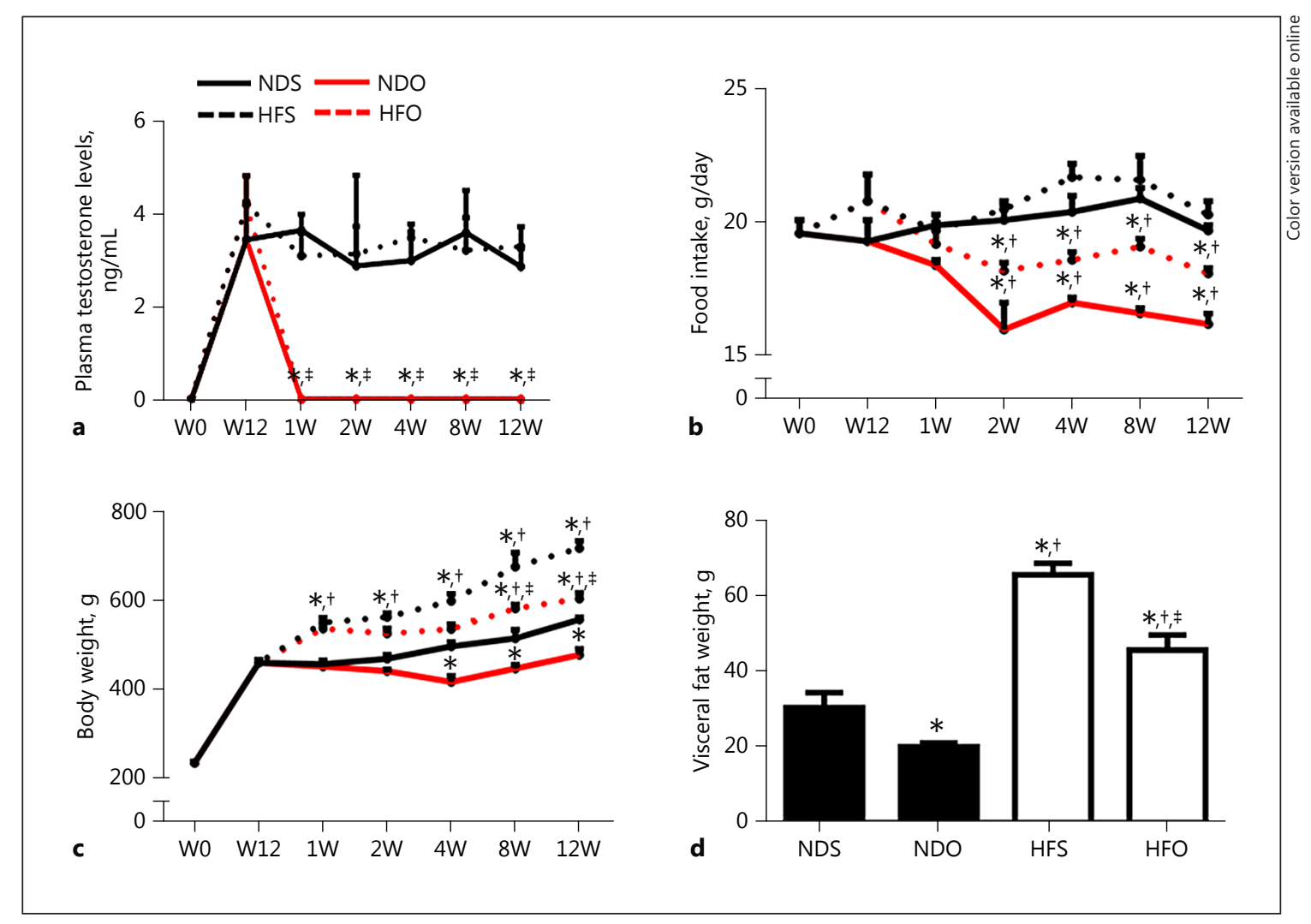

Fig. 1. Effects of testosterone deprivation on peripheral metabolic parameters ( $n=8 /$ group). a Plasma testosterone levels. b Daily food intake. c Body weight. $\mathbf{d}$ Visceral fat weight. ${ }^{*} p<0.05$ versus NDS; ${ }^{\dagger} p<0.05$ versus NDO; ${ }^{\ddagger} p<0.05$ versus HFS. NDS, normal diet-fed rats and sham operation; NDO, normal diet-fed rats with orchiectomy; HFS, high-fat diet-fed rats with sham operation; HFO, high-fat diet-fed rats with orchiectomy.

including PGC1a (1:200 dilution; Santa Cruz), CPT1 (1:1,000 dilution; Santa Cruz), OXPHOS (1:1,000 dilution; Abcam), p-Drp1 $1^{\text {ser616 }}$ (1:1,000 dilution), Drp1 (1:1,000 dilution), Mfn2 (1:1,000 dilution), andOPA1 (1:1,000 dilution; all Cell Signaling).

For apoptosis determination, membranes were incubated with primary antibodies including Bax (1:200 dilution; Santa Cruz), Bcl2 (1:1,000 dilution; Abcam), cleaved caspase 3 (1:1,000 dilution; Cell Signaling) and caspase 3 (1:1,000 dilution; Cell Signaling).

For myocardial insulin signaling determination, membranes were incubated with primary antibodies including $\mathrm{p}-\mathrm{IR}^{\mathrm{tyr} 1162 / 1163}$ (1:200 dilution; Santa Cruz), IR (1:200 dilution; Santa Cruz), p$\mathrm{Akt}^{\mathrm{ser} 473}$ (1:1,000 dilution; Cell Signaling) and Akt (1:1,000 dilution; Cell Signaling).

After incubation with primary antibodies at $4{ }^{\circ} \mathrm{C}$ overnight, membranes were transferred to secondary antibodies. They were then exposed to an enhanced chemiluminescent substrate (Clarity Western ECL Substrate; Bio-Rad). The Western blot images and densitometric analysis were carried out using the ChemiDoc Imaging system with Image Lab software (Bio-Rad) [16, 18].

Statistical Analysis

Data are presented as mean \pm SE. All statistical analyses were performed using GraphPad 6.0. For time course analysis, data were analyzed using a two-way ANOVA followed by a Tukey post hoc test. For single time point analysis, data were analyzed using a oneway ANOVA followed by a Tukey post hoc test. $p<0.05$ was considered statistically significant.

\section{Results}

\section{Testosterone Deprivation Did Not Aggravate}

Metabolic Impairment in Obese Insulin-Resistant Rats

At baseline there was no difference in body weight, food intake, plasma glucose, insulin, HOMA index, lipid profiles, and plasma testosterone levels between ND- and HFD-fed rats (Fig. 1b, c; online suppl. Fig. 2, 3). Plasma testosterone levels were increased in both the ND and HFD groups at week 12 (Fig. 2a).

After ORX, plasma testosterone levels were decreased in both NDO and HFO rats, compared with NDS and HFS groups (Fig. 1a). Body weight and food intake were 


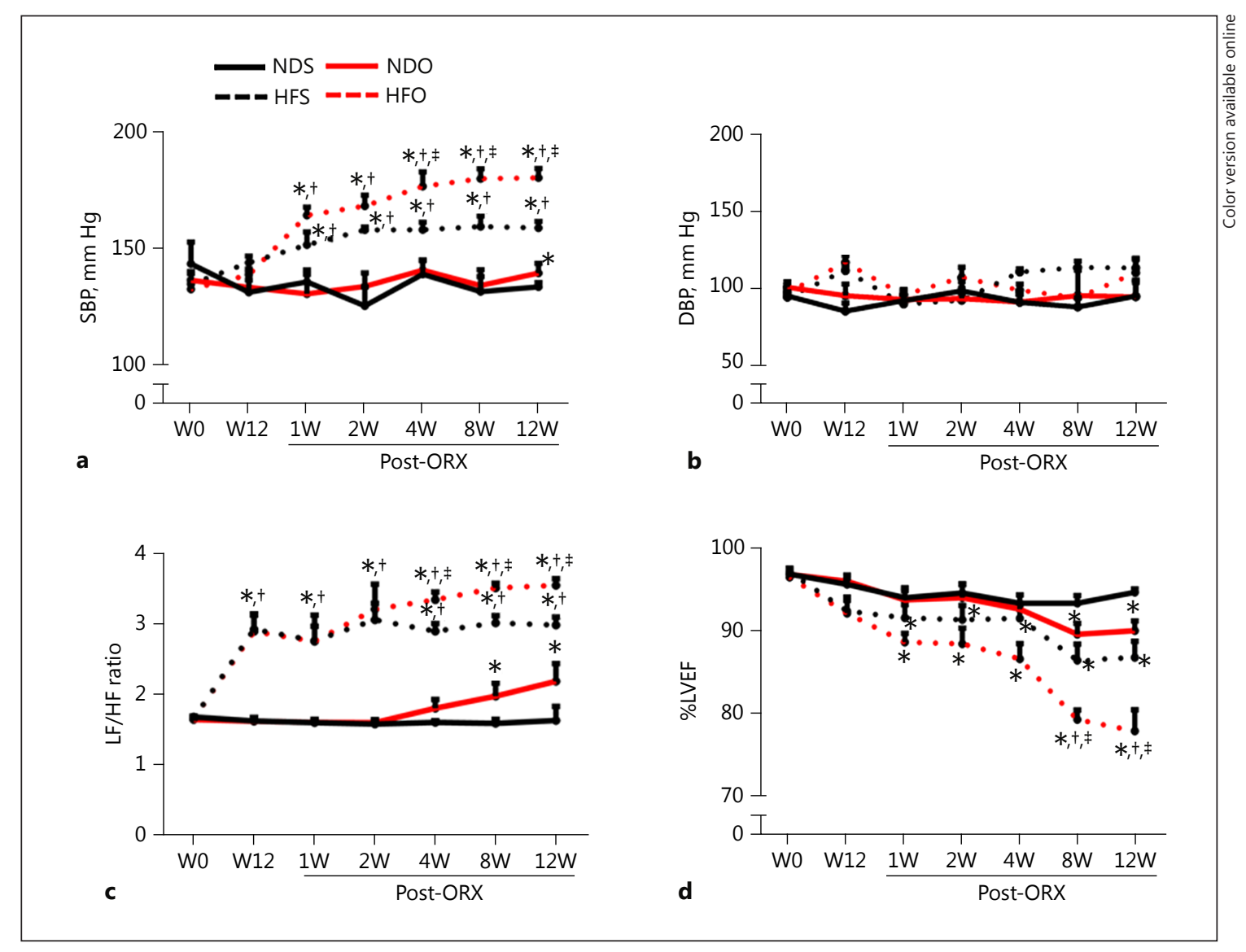

Fig. 2. Effects of testosterone deprivation on blood pressure, cardiac autonomic balance, and LV function in normal and obese insulin-resistant rats ( $n=8$ /group). a SBP. b DBP. c LF/HF ratio. d \%LVEF. ${ }^{*} p<0.05$ versus NDS; ${ }^{\dagger} p<0.05$ versus NDO; ${ }^{\ddagger} p<0.05$ versus HFS. NDS, normal diet-fed rats and sham operation; NDO, normal dietfed rats with orchiectomy; HFS, high-fat diet-fed rats with sham operation; HFO, high-fat diet-fed rats with orchiectomy; SBP, systolic blood pressure; DBP, diastolic blood pressure; LF/HF ratio: low-frequency/highfrequency ratio of heart rate variability; \%LVEF, percentage of left-ventricular ejection fraction.

reduced in both NDO and HFO groups, compared with NDS and HFS groups (Fig. 1b, c). Visceral fat weight was determined at 12 weeks post-ORX, and our data showed that it was reduced in both NDO and HFO groups, compared with NDS and HFS groups (Fig. 1d).

All rats fed on HFD for 12 weeks developed obesityrelated insulin resistance. At 1, 2, 4, 8, and 12 weeks after ORX, all metabolic parameters including plasma glucose, insulin levels, HOMA-IR, and lipid profiles showed no difference from baseline in NDO and HFO groups, compared with NDS and HFS groups (see online suppl. Fig. 2, 3 ), suggesting that testosterone deprivation did not alter peripheral insulin resistance in both ND- and HFD-fed rats.

Lack of Testosterone and Heart Dysfunction in Obese Rats
Testosterone Deprivation Aggravated LV Dysfunction in Obese Insulin-Resistant Rats

At baseline, SBP, DBP, LF/HF ratio, and \%LVEF were no different between groups. After 12 weeks of HFD feeding, $\mathrm{LF} / \mathrm{HF}$ ratio was increased and \%LVEF was decreased in both HFS and HFO groups, compared with NDS and NDO groups (Fig. 2a-d). However, there was no difference in SBP and DBP between ND- and HFD-fed rats (Fig. 2a-d).

After ORX, in the NDO group, SBP was increased at 12 weeks post-ORX, LF/HF ratio was increased at 8 and 12 weeks post-ORX, and \%LVEF was reduced at 8 and 12 weeks post-ORX, compared with the NDS group (Fig. 2a-d). In the HFO group, SBP was markedly increased at 4,8 , and 12 weeks post-ORX, LF/HF ratio was 
Table 1. Effects of testosterone deprivation on invasive left-ventricular function parameters in ND- and HFD-fed rats

\begin{tabular}{lcccc}
\hline Parameters & NDS & NDO & HFS & HFO \\
\hline $\mathrm{HR}, \mathrm{bpm}$ & $331 \pm 48$ & $344 \pm 35$ & $450 \pm 27^{*, \dagger}$ & $448 \pm 32^{*, \dagger}$ \\
$\mathrm{ESP}, \mathrm{mm} \mathrm{Hg}$ & $130 \pm 5$ & $115 \pm 3^{*}$ & $112 \pm 7^{*, \dagger}$ & $115 \pm 10^{*, \dagger}$ \\
$\mathrm{EDP}, \mathrm{mm} \mathrm{Hg}$ & $6 \pm 2$ & $5 \pm 2$ & $14 \pm 3^{*, \dagger}$ & $21 \pm 5^{*, \dagger}, \neq$ \\
$+\mathrm{dP} / \mathrm{dt}, \mathrm{mm} \mathrm{Hg} / \mathrm{s}$ & $8,832 \pm 368$ & $8,732 \pm 412$ & $6,131 \pm 212^{*, \dagger}$ & $6,014 \pm 107^{*, \dagger}$ \\
$-\mathrm{dP} / \mathrm{dt}, \mathrm{mm} \mathrm{Hg} / \mathrm{s}$ & $-5,307 \pm 213$ & $-5,136 \pm 347$ & $3,341 \pm 475^{*, \dagger}$ & $2,987 \pm 351^{*, \dagger, \ddagger}$ \\
$\mathrm{ESV}, \mu \mathrm{L}$ & $20 \pm 2$ & $18 \pm 4$ & $24 \pm 7^{*, \dagger}$ & $25 \pm 6^{*, \dagger}$ \\
$\mathrm{EDV}, \mu \mathrm{L}$ & $322 \pm 35$ & $335 \pm 41$ & $411 \pm 31^{*, \dagger}$ & $432 \pm 32^{*, \dagger, \ddagger}$ \\
$\mathrm{SV}, \mu \mathrm{L} / \mathrm{g}$ & $0.5 \pm 0.04$ & $0.6 \pm 0.02$ & $0.32 \pm 0.05^{*, \dagger}$ & $0.35 \pm 0.06^{*, \dagger}$ \\
$\% \mathrm{LVEF}$ & $96.8 \pm 1.4$ & $92.1 \pm 0.4^{*}$ & $87 \pm 2.35^{*, \dagger}$ & $78 \pm 2.25^{*, \dagger, \ddagger}$ \\
\hline
\end{tabular}

ND, normal diet; HFD, high-fat diet; NDS, normal diet-fed rats and sham operation; NDO, normal diet-fed rats with orchiectomy; HFS, high-fat diet-fed rats with sham operation; HFO, high-fat diet-fed rats with orchiectomy; HR, heart rate; ESP, end-systolic pressure; EDP, end-diastolic pressure; ESV, end-systolic volume; EDV, end-diastolic volume; SV, stroke volume; \%LVEF, percentage of left-ventricular ejection fraction. ${ }^{*} \mathrm{p}<0.05$ versus NDS; $\uparrow \mathrm{p}<0.05$ versus NDO; $\neq \mathrm{p}<0.05$ versus HFS.

increased at 4, 8, and 12 weeks post-ORX, and \%LVEF was reduced at 8 and 12 weeks post-ORX, compared with the HFS group (Fig. 2a-d).

In addition, LV function was determined invasively at 12 weeks post-ORX. In the NDO group, ESP and \%LVEF were lower than in the NDS group, indicating LV dysfunction (Table 1). In the HFS group, HR, EDP, ESV, and EDV were increased, and ESP, $\pm \mathrm{dP} / \mathrm{dt}, \mathrm{SV}$, and \%LVEF were decreased, compared with the NDS group, again indicating LV dysfunction (Table 1). In the HFO group, EDP and EDV were markedly increased, and $-\mathrm{dP} / \mathrm{dt}$ and \%LVEF were markedly decreased, compared with the HFS group (Table 1). These data suggested that testosterone deprivation aggravated LV dysfunction in HFDfed rats.

\section{Testosterone Deprivation Aggravated Cardiac}

Mitochondrial Dysfunction in Obese Insulin-Resistant

Rats

Our data showed that cardiac mitochondrial ROS levels were higher in the NDO group than in the NDS group, whereas MMP changes and mitochondrial swelling were no different between these two groups (Fig. 3a-c). In the HFS group, mitochondrial ROS levels and mitochondrial swelling were increased, and MMP changes were decreased, compared with the NDS group (Fig. $3 \mathrm{a}-\mathrm{c}$ ). In the HFO group, mitochondrial ROS and mitochondrial swelling were markedly increased, and MMP changes were markedly decreased, compared with the HFS group
(Fig. 3a-c). Representative images of cardiac mitochondria morphology of rats in the NDS, NDO, HFS, and HFO groups are shown in Figure 3e. In addition, our data demonstrated that state 3 respiration was decreased in NDO rats, compared with NDS rats. In HFD groups, HFS and HFO rats had lower state 3 respiration than NDS rats, and HFO rats had the lowest state 3 respiration, compared with NDS, NDO, and HFS rats (Fig. 3d). State 4 respiration was decreased in both HFS and HFO rats, compared with NDS and NDO rats. However, state 4 respiration was not different between HFS and HFO rats. These data suggested that testosterone deprivation aggravated cardiac mitochondrial dysfunction by decreasing ADP-stimulated mitochondrial respiration in obese insulin-resistant rats.

Testosterone Deprivation Aggravated Circulating and Cardiac Oxidative Stress in Obese Insulin-Resistant Rats

When considering the results from the ND-fed rats, plasma and cardiac MDA were increased in NDO rats, compared with these parameters in the NDS rats (see online suppl. Fig. 5a, b). In HFD-fed rats, our data showed that plasma and cardiac MDA were higher in HFS rats than in both NDS and NDO rats, and also that these parameters were markedly increased in HFO rats, compared with HFS rats (Fig. 4a, b), suggesting that testosterone deprivation aggravated circulating and cardiac oxidative stress in both ND- and HFD-fed rats. 


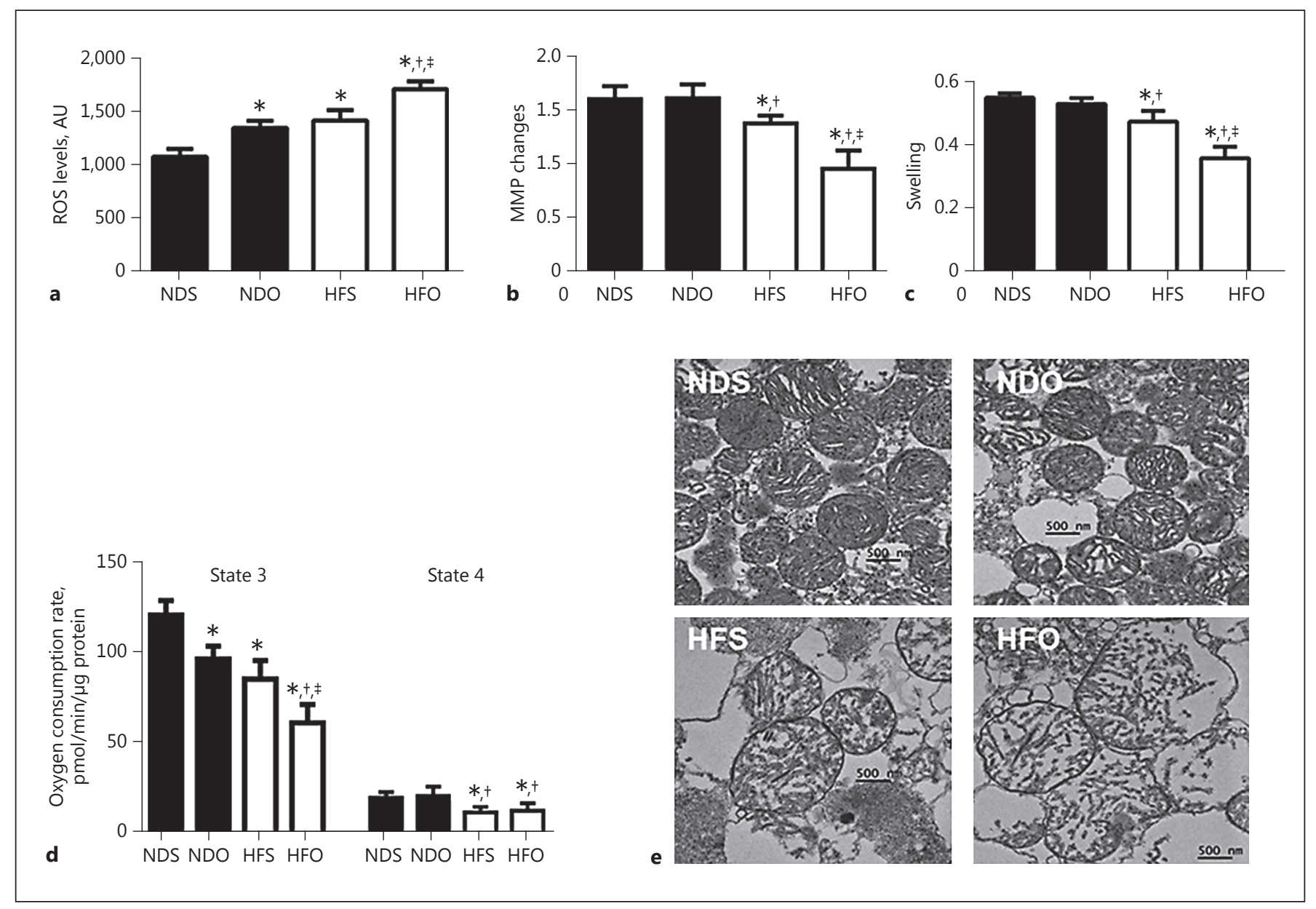

Fig. 3. Effects of testosterone deprivation on cardiac mitochondrial function in normal and obese insulin-resistant rats $(n=8$ / group). a ROS levels. b MMP changes. c Swelling. d Cardiac mitochondrial oxygen consumption rate. e Representative images of cardiac mitochondria. ${ }^{*} p<0.05$ versus NDS; ${ }^{\dagger} p<0.05$ versus

Testosterone Deprivation Aggravated the Reduction of Mitochondrial Respiratory Complex and Mitochondrial Fusion Proteins, and Aggravated Cardiac Apoptosis, but Did Not Alter Myocardial Insulin Receptor Function in Obese Insulin-Resistant Rats

In ND-fed rats, PGC1 $a, \mathrm{CPT} 1$, mitochondrial respiratory complex I-V, p-Drp1 $1^{\text {ser616}} / \mathrm{Drp} 1, \mathrm{Mfn} 2$, and OPA1 protein levels were no different between NDS and NDO groups (Fig. 4a-d; online suppl. Fig. 5a, b). In the HFS group, CPT1, mitochondrial respiratory complex I, III, and V, Mfn2, and OPA1 protein levels were decreased, compared with the NDS and NDO groups (Fig. 4b-d; online suppl. Fig. 5b). In the HFO group, PGC1a, mitochondrial respiratory complex I and III, Mfn2, and OPA1 pro-
NDO; ${ }^{*} p<0.05$ versus HFS. NDS, normal diet-fed rats and sham operation; NDO, normal diet-fed rats with orchiectomy; HFS, high-fat diet-fed rats with sham operation; HFO, high-fat diet-fed rats with orchiectomy; ROS, reactive oxygen species; MMP, mitochondrial membrane potential. tein levels were markedly decreased, compared with the HFS group (Fig. 4b-c; online suppl. Fig. 5a). However, there was no difference in $\mathrm{CPT} 1$ and complex $\mathrm{V}$ protein levels between the HFS and HFO groups (Fig. 4c; online suppl. Fig. 5b). Moreover, p-Drp1 $1^{\text {ser616}} / \mathrm{Drp} 1$ protein levels were not different among groups (Fig. 4a).

As regards cardiac apoptosis in ND-fed rats, there was no difference in $\mathrm{Bax}, \mathrm{Bcl} 2$, and cleaved caspase 3/caspase 3 protein levels between NDS and NDO groups (see online suppl. Fig. 5c, d). However, HFS and HFO groups had decreased $\mathrm{Bcl} 2$ protein levels, whereas Bax and cleaved caspase $3 /$ caspase 3 protein levels were not different in the NDS, NDO, and HFS groups (see online suppl. Fig. 5c, d). In the HFO group, cleaved caspase 3/caspase 3 protein levels were increased when compared with the 


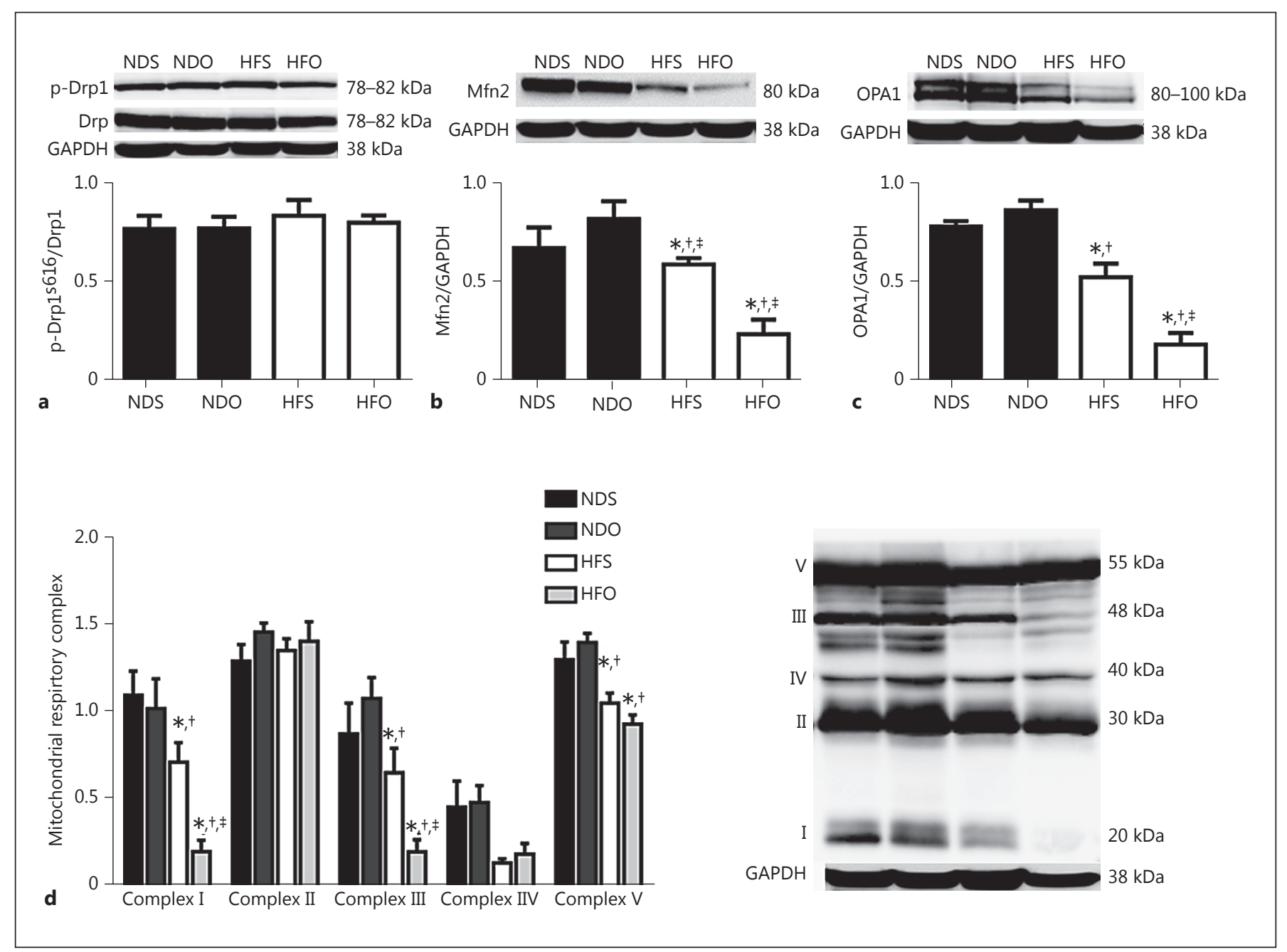

Fig. 4. Effects of testosterone deprivation on mitochondrial dynamics and respiratory complex in normal and obese insulin-resistant rats $\left(n=8 /\right.$ group). a $\mathrm{p}$-Drp $1^{\text {ser616}} / \mathrm{Drp} 1$. b Mfn2 protein levels. c OPA1 protein levels. d Mitochondrial respiratory complex I-V protein levels. ${ }^{*} p<0.05$ versus NDS; ${ }^{\dagger} p<0.05$ versus NDO;
${ }^{\ddagger} p<0.05$ versus HFS. NDS, normal diet-fed rats with sham operation; NDO, normal diet-fed rats with orchiectomy; HFS, high-fat diet-fed rats with sham operation; HFO, high-fat diet-fed rats with orchiectomy; Drp1, dynamic-related protein1; Mfn2, mitofusin2; OPA1, optic atrophy1.
HFS group (see online suppl. Fig. 5e). However, there was no difference in the $\mathrm{Bax}$ and $\mathrm{Bcl} 2$ proteins levels between HFS and HFO groups (see online suppl. Fig. 5c, d).

Myocardial insulin signaling, in ND-fed rats, $\mathrm{p}$ -

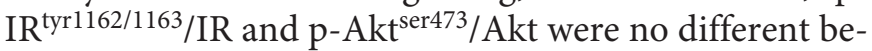
tween NDS and NDO groups (see online suppl. Fig. 6). In HFD-fed rats, $\mathrm{p}-\mathrm{IR}^{\mathrm{tyr} 1162 / 1163} / \mathrm{IR}$ and $\mathrm{p}-\mathrm{Akt}^{\mathrm{ser} 473} / \mathrm{Akt}$ were decreased in both HFS and HFO groups, compared with NDS and NDO groups (see online suppl. Fig. 6). How-

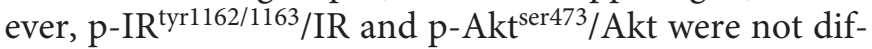
ferent between HFS and HFO groups (see online suppl. Fig. 6).

\section{Discussion}

Our study demonstrated the following: (i) in obese insulin-resistant rats testosterone deprivation reduced body weight, did not aggravate peripheral insulin response, but caused a decrease in myocardial insulin signaling; (ii) in obese insulin-resistant rats testosterone deprivation aggravated LV dysfunction and as there was, as a result, increased cardiac sympathovagal imbalance, increased systolic blood pressure, deterioration in cardiac mitochondrial dysfunction, reduced mitochondrial respiratory complex I and III protein levels, reduced mito- 
chondrial fusion protein levels, and increased myocardial apoptosis; and (iii) in normal rats, testosterone deprivation caused cardiac sympathovagal imbalance and LV dysfunction via increased oxidative stress.

In this study, testosterone deprivation led to a reduction in body weight due to reduced food intake in both normal and obese insulin-resistant rats. Previous studies have demonstrated that circulating adiponectin levels were increased in both ND- and HFD-fed rodents with testosterone deprivation [22, 23]. Adiponectin is known to promote weight loss by increasing energy expenditure [24] and reducing appetite [25]. Although body weight was reduced following testosterone deprivation, the peripheral insulin-resistant condition was not affected in both normal and obese insulin-resistant rats. In addition, testosterone deprivation did not aggravate the impairment of myocardial insulin receptor function in these obese insulin-resistant rats. Our findings are inconsistent with a previous study by Donner et al. [12] which showed that testosterone deprivation, after the onset of obesityrelated insulin resistance, aggravated the insulin-resistant condition in male rats. The discrepancy could be due to the difference in the diet used in that study to create the obese insulin-resistant model, and the duration of testosterone deprivation. In that study, they used a high-fat and high-sucrose (HFD/HS) diet to induce obesity-related insulin resistance, and metabolic parameters were determined at 20 weeks post-ORX [12] whilst we used HFD to induce obesity-related insulin resistance, and metabolic parameters were determined at 12 weeks post-ORX.

We previously demonstrated that testosterone deprivation in very young rats accelerated but did not aggravate LV dysfunction in obese insulin-resistant rats [4]. In this study, we found that testosterone deprivation during adulthood aggravated LV dysfunction and cardiac sympathovagal imbalance in obese insulin-resistant rats. Therefore, our data suggested that an alteration in LV function in obese insulin-resistant rats with testosterone deprivation depends upon the age at which testosterone deprivation occurs.

In this study, the potential mechanism responsible for the deleterious effects on LV function was determined. In normal rats subjected to testosterone deprivation, our data showed that systemic and cardiac oxidative stress levels were increased, together with an increase in cardiac mitochondrial ROS level when compared with normal rats without testosterone deprivation. However, cardiac MMP and mitochondrial swelling were within normal limits. Excessive levels of systemic and mitochondrial oxidative stress were found to stimulate central and periph-

Lack of Testosterone and Heart

Dysfunction in Obese Rats eral sympathetic activity [26-28]. This abnormal activity was followed by cardiac autonomic imbalance, contributing to LV dysfunction [29] (see online suppl. Fig. 7).

Cardiac mitochondrial membrane depolarization and cardiac mitochondrial swelling were observed in obese insulin-resistant rats, but not in normal rats with testosterone deprivation, suggesting more severe cardiac mitochondrial dysfunction and mitochondrial damage. It is interesting that testosterone deprivation led to increased mitochondrial ROS levels, and aggravated cardiac mitochondrial membrane depolarization and swelling in obese insulin-resistant rats. These data suggested that testosterone deprivation aggravated cardiac mitochondrial damage in obese insulin-resistant rats.

After mitochondrial damage, the adaptation of mitochondrial dynamics is usually activated by increasing mitochondrial fission and fusion proteins [30, 31]. Mitochondrial fission helps to remove damaged mitochondria via the mitophagy mechanism [32]. In addition, the process of mitochondrial fusion enhances mitochondrial oxidative phosphorylation by upregulating activity of the mitochondrial respiratory complex [32]. However, in obese insulin-resistant rats with testosterone deprivation, mitochondrial fusion protein was reduced, as indicated by reduced Mfn 2 and OPA1 proteins, whereas mitochondrial fission protein was not altered, when compared with their sham operation group. A reduction in Mfn2 protein results in reduced outer mitochondrial membrane fusion [33]. Previous studies demonstrated that silencing the Mfn2 increased sensitivity to apoptotic stimuli, and accelerated cytochrome $\mathrm{c}$ release [33]. Thus, our data suggested that testosterone deprivation reduced Mfn2 protein levels, which may lead to reduced outer mitochondrial membrane fusion, and promoted cardiac apoptosis as shown in these testosterone-deprived obese insulinresistant rats (Fig. 4).

OPA1 is a marker for inner mitochondrial membrane fusion [34], which is responsible for maintaining mitochondrial cristae shape [34], and cristae are the critical site for the oxidative phosphorylation process [35]. Therefore, a reduction in OPA1 directly affects mitochondrial respiration via a reduction in the oxidative phosphorylation process [36]. In the obese insulin-resistant rats with testosterone deprivation, OPA 1 protein levels were reduced along with a reduction in mitochondrial respiratory complexes I and III. Thus, our results suggest that testosterone deprivation reduced OPA1 protein, which possibly leads to reduced inner mitochondrial membrane fusion. Furthermore, we also found that testosterone deprivation reduced $\mathrm{PGC1}$ a protein expression 
in obese insulin-resistant rats. As PGC1 a mediates mitochondrial biogenesis and the oxidative phosphorylation process [35-38], these findings suggested that testosterone deprivation could reduce mitochondrial biogenesis and oxidative phosphorylation in obese insulin-resistant rats.

Although testosterone deprivation did not affect mitochondrial OXPHOS protein levels in normal rats, it decreased ADP-stimulated cardiac mitochondrial oxygen consumption. These data suggested that in normal rats, cardiac mitochondrial dysfunction occurred following testosterone deprivation, and preceded the reduction of OXPHOS protein levels. In obese insulin-resistant rats, testosterone deprivation aggravated cardiac mitochondrial dysfunction since it markedly decreased ADP-stimulated cardiac mitochondrial oxygen consumption and reduced complex I and III protein levels. However, testosterone deprivation did not affect state 4 respiration. Our data also showed that testosterone deprivation did not alter complex $\mathrm{V}$ protein levels in both normal rats and obese insulin-resistant rats.
In conclusion, our findings demonstrated that testosterone deprivation aggravated LV dysfunction in obese insulin-resistant rats by exacerbating cardiac mitochondrial dysfunction and decreasing mitochondrial fusion proteins, reducing mitochondrial oxidative phosphorylation proteins, and increasing apoptosis. However, these adverse cardiac effects are independent of the status of both peripheral and myocardial insulin signaling.

\section{Acknowledgments}

This work was supported by Thailand Research Fund grants TRF-RTA6080003 (S.C.C.), TRG6080005 (N.A.), and the Royal Golden Jubilee Program (T.C. and S.C.C.), an NSTDA Research Chair grant from the National Science and Technology Development Agency Thailand (N.C.), and Faculty of Medicine, Chiang Mai University Endowment Fund 131/2559 (N.A.).

\section{Disclosure Statement}

The authors declare that there are no conflicts of interest.

\section{References}

-1 Cattabiani C, Basaria S, Ceda GP, Luci M, Vignali A, Lauretani F, Valenti G, Volpi R, Maggio M: Relationship between testosterone deficiency and cardiovascular risk and mortality in adult men. J Endocrinol Invest 2012;35: 104-120.

- Poliwczak AR, Tylinska M, Broncel M: Effect of short-term testosterone replacement therapy on heart rate variability in men with hypoandrogen-metabolic syndrome. Pol Arch Med Wewn 2013;123:467-473.

-3 Kang NN, Fu L, Xu J, Han Y, Cao JX, Sun JF, Zheng M: Testosterone improves cardiac function and alters angiotensin II receptors in isoproterenol-induced heart failure. Arch Cardiovasc Dis 2012;105:68-76.

-4 Pongkan W, Pintana H, Sivasinprasasn S, Jaiwongkam T, Chattipakorn SC, Chattipakorn $\mathrm{N}$ : Testosterone deprivation accelerates cardiac dysfunction in obese male rats. J Endocrinol 2016;229:209-220.

5 Witayavanitkul N, Woranush W, Bupha-Intr $\mathrm{T}$, Wattanapermpool J: Testosterone regulates cardiac contractile activation by modulating SERCA but not NCX activity. Am J Physiol Heart Circ Physiol 2013;304:H465H472.

6 Rovira-Llopis S, Banuls C, de Maranon AM, Diaz-Morales N, Jover A, Garzon S, Rocha M, Victor VM, Hernandez-Mijares A: Low tes- tosterone levels are related to oxidative stress, mitochondrial dysfunction and altered subclinical atherosclerotic markers in type 2 diabetic male patients. Free Radic Biol Med 2017; 108:155-162.

7 Ong SB, Hausenloy DJ: Mitochondrial morphology and cardiovascular disease. Cardiovasc Res 2010;88:16-29.

-8 Wang F, Yang J, Sun J, Dong Y, Zhao H, Shi H, Fu L: Testosterone replacement attenuates mitochondrial damage in a rat model of myocardial infarction. J Endocrinol 2015;225:101-111.

-9 Duvezin-Caubet S, Jagasia R, Wagener J, Hofmann S, Trifunovic A, Hansson A, Chomyn A, Bauer MF, Attardi G, Larsson NG, Neupert W, Reichert AS: Proteolytic processing of OPA1 links mitochondrial dysfunction to alterations in mitochondrial morphology. J Biol Chem 2006;281:37972-37979.

10 Suen DF, Norris KL, Youle RJ: Mitochondrial dynamics and apoptosis. Genes Dev 2008;22: $1577-1590$

11 Fui MN, Dupuis P, Grossmann M: Lowered testosterone in male obesity: mechanisms, morbidity and management. Asian J Androl 2014;16:223-231.

12 Donner DG, Elliott GE, Beck BR, Bulmer AC, $\mathrm{Du}$ Toit EF: Impact of diet-induced obesity and testosterone deficiency on the cardiovascular system: a novel rodent model represen- tative of males with testosterone-deficient metabolic syndrome (TDMetS). PLoS One 2015;10:e 0138019.

13 Donner DG, Elliott GE, Beck BR, Bulmer AC, Lam AK, Headrick JP, Du Toit EF: Trenbolone improves cardiometabolic risk factors and myocardial tolerance to ischemia-reperfusion in male rats with testosterone-deficient metabolic syndrome. Endocrinology 2016; 157:368-381.

14 Pratchayasakul W, Kerdphoo S, Petsophonsakul P, Pongchaidecha A, Chattipakorn N, Chattipakorn SC: Effects of high-fat diet on insulin receptor function in rat hippocampus and the level of neuronal corticosterone. Life Sci 2011;88:619-627.

-15 Ouwens DM, Boer C, Fodor M, de Galan P, Heine RJ, Maassen JA, Diamant M: Cardiac dysfunction induced by high-fat diet is associated with altered myocardial insulin signalling in rats. Diabetologia 2005;48:1229-1237.

-16 Apaijai N, Inthachai T, Lekawanvijit S, Chattipakorn SC, Chattipakorn N: Effects of dipeptidyl peptidase-4 inhibitor in insulin-resistant rats with myocardial infarction. J Endocrinol 2016;229:245-258.

17 Chattipakorn N, Incharoen T, Kanlop N, Chattipakorn S: Heart rate variability in myocardial infarction and heart failure. Int J Cardiol 2007;120:289-296.
10

Gerontology

DOI: $10.1159 / 000487188$
Apaiajai/Chunchai/Jaiwongkam/

Kerdphoo/Chattipakorn/Chattipakorn 
18 Tunapong W, Apaijai N, Yasom S, Tanajak P, Wanchai K, Chunchai T, Kerdphoo S, Eaimworawuthikul S, Thiennimitr P, Pongchaidecha A, Lungkaphin A, Pratchayasakul W, Chattipakorn SC, Chattipakorn N: Chronic treatment with prebiotics, probiotics and synbiotics attenuated cardiac dysfunction by improving cardiac mitochondrial dysfunction in male obese insulin-resistant rats. Eur J Nutr 2017, Epub ahead of print.

-19 Apaijai N, Pintana H, Chattipakorn SC, Chattipakorn N: Effects of vildagliptin versus sitagliptin, on cardiac function, heart rate variability and mitochondrial function in obese insulin-resistant rats. Br J Pharmacol 2013; 169:1048-1057.

20 Aon MA, Stanley BA, Sivakumaran V, Kembro JM, O'Rourke B, Paolocci N, Cortassa S: Glutathione/thioredoxin systems modulate mitochondrial $\mathrm{H}_{2} \mathrm{O}_{2}$ emission: an experimental-computational study. J Gen Physiol 2012; 139:479-491.

21 Rogers GW, Brand MD, Petrosyan S, Ashok D, Elorza AA, Ferrick DA, Murphy AN: High throughput microplate respiratory measurements using minimal quantities of isolated mitochondria. PLoS One 2011;6:e21746.

-22 Harada N, Hanaoka R, Hanada K, Izawa T, Inui $\mathrm{H}$, Yamaji R: Hypogonadism alters cecal and fecal microbiota in male mice. Gut Microbes 2016;7:533-539.

-23 Yarrow JF, Beggs LA, Conover CF, McCoy SC, Beck DT, Borst SE: Influence of androgens on circulating adiponectin in male and female rodents. PLoS One 2012;7:e47315.
24 Dridi S, Taouis M: Adiponectin and energy homeostasis: consensus and controversy. J Nutr Biochem 2009;20:831-839.

25 Bassi M, do Carmo JM, Hall JE, da Silva AA: Chronic effects of centrally administered adiponectin on appetite, metabolism and blood pressure regulation in normotensive and hypertensive rats. Peptides 2012;37:1-5

26 Campese VM, Ye S, Zhong H, Yanamadala V, Ye Z, Chiu J: Reactive oxygen species stimulate central and peripheral sympathetic nervous system activity. Am J Physiol Heart Circ Physiol 2004;287:H695-H703.

27 Andersson DC, Fauconnier J, Yamada T, Lacampagne A, Zhang SJ, Katz A, Westerblad $\mathrm{H}$ : Mitochondrial production of reactive oxygen species contributes to the beta-adrenergic stimulation of mouse cardiomyocytes. J Physiol 2011;589:1791-1801.

28 Ye S, Zhong H, Yanamadala S, Campese VM: Oxidative stress mediates the stimulation of sympathetic nerve activity in the phenol renal injury model of hypertension. Hypertension 2006;48:309-315.

29 Triposkiadis F, Karayannis G, Giamouzis G, Skoularigis J, Louridas G, Butler J: The sympathetic nervous system in heart failure physiology, pathophysiology, and clinical implications. J Am Coll Cardiol 2009;54:1747-1762.

30 Westermann B: Bioenergetic role of mitochondrial fusion and fission. Biochim Biophys Acta 2012;1817:1833-1838.

31 Kluge MA, Fetterman JL, Vita JA: Mitochondria and endothelial function. Circ Res 2013; 112:1171-1188

32 Westermann B: Mitochondrial fusion and fission in cell life and death. Nat Rev Mol Cell Biol 2010;11:872-884.
33 Weaver D, Eisner V, Liu X, Varnai P, Hunyady L, Gross A, Hajnoczky G: Distribution and apoptotic function of outer membrane proteins depend on mitochondrial fusion. Mol Cell 2014;54:870-878.

34 Hall AR, Burke N, Dongworth RK, Hausenloy DJ: Mitochondrial fusion and fission proteins: novel therapeutic targets for combating cardiovascular disease. Br J Pharmacol 2014; 171:1890-1906.

35 Perciavalle RM, Stewart DP, Koss B, Lynch J, Milasta S, Bathina M, Temirov J, Cleland MM, Pelletier S, Schuetz JD, Youle RJ, Green DR, Opferman JT: Anti-apoptotic MCL-1 localizes to the mitochondrial matrix and couples mitochondrial fusion to respiration. Nat Cell Biol 2012;14:575-583.

-36 Van Bergen NJ, Crowston JG, Kearns LS, Staffieri SE, Hewitt AW, Cohn AC, Mackey DA, Trounce IA: Mitochondrial oxidative phosphorylation compensation may preserve vision in patients with OPA1-linked autosomal dominant optic atrophy. PLoS One 2011; 6:e21347.

37 LeBleu VS, O’Connell JT, Gonzalez Herrera KN, Wikman H, Pantel K, Haigis MC, de Carvalho FM, Damascena A, Domingos Chinen LT, Rocha RM, Asara JM, Kalluri R: PGC-1a mediates mitochondrial biogenesis and oxidative phosphorylation in cancer cells to promote metastasis. Nat Cell Biol 2014;16:9921003, 1001-1015.

38 Liang H, Ward WF: PGC-1 $1 \alpha$ : a key regulator of energy metabolism. Adv Physiol Educ 2006;30:145-151.
Lack of Testosterone and Heart

Dysfunction in Obese Rats 